\title{
Paracetamol: Risikofaktor für Asthma
}

\author{
Noch längst sind nicht alle Einflussfaktoren bekannt, die zur klinischen \\ Manifestation eines Asthma bronchiale beitragen. Im Rahmen der \\ ISAAC-Studie wurde jetzt der Zusammenhang zwischen der Einnahme \\ von Paracetamol und dem Asthmarisiko bei Kindern deutlich.
}

\begin{abstract}
n der Pädiatrie stand Paracetamol in den 1950er Jahren zunächst als Ersatz für das nephrotoxische Analgetikum Phenacetin zur Verfügung, seit 1985 ist es für Kinder Antipyretikum und Analgetikum der ersten Wahl. Dass die Substanz das Risiko für die Entwicklung eines Asthmas erhöht, wird schon seit längerem angenommen. Um diese Hypothese zu untersuchen, wurden Daten von Kindern im Alter zwischen sechs und sieben Jahren analysiert. Die Untersuchung war Teil der International Study of Asthma and Allergies in Childhood (ISAAC, Phase 3), einer multizentrischen bevölkerungsbasierten Querschnittsuntersuchung zu
\end{abstract}

allergologischen Fragestellungen bei Schulkindern.

205.487 Kinder in 31 Ländern wurden in die Analyse einbezogen. Es ergab sich ein signifikantes und etwa um $50 \%$ erhöhtes Risiko für Asthma im Alter von sechs bis sieben Jahren nach Gabe von Paracetamol im ersten Lebensjahr (Odds Ratio: 1,46). Auch eine Einnahme des Medikaments innerhalb des gerade vergangenen Jahres war mit einer signifikant erhöhten Wahrscheinlichkeit für Asthma assoziiert - und zwar in Abhängigkeit von der Dosierung: Wurde Paracetamol mindestens einmal pro Jahr eingenommen, ergab sich eine Odds Ratio von 1,61, die Verabreichung mindestens einmal pro Monat verdreifachte das Risiko (Odds Ratio: 3,23). Eine Korrelation bestand auch zwischen Paracetamolanwendung und dem Risiko für schwere Asthmasymptome (22\% bei mittlerer Einnahmefrequenz und $38 \%$ bei häufiger Gabe). Außerdem war eine Paracetamolmedikation in den ersten Lebensjahren mit einem erhöhten Risiko für Rhinokonjunktivitis und Ekzem verknüpft.

Fazit: Die Einnahme von Paracetamol während der ersten Lebensjahre ist mit einem erhöhten Risiko für Asthma, Rhinokonjunktivitis und Ekzem assoziiert. Eine Paracetamolmedikation im Kindesalter dürfte damit als Asthmarisikofaktor einzustufen sein.

\section{$b k$}

\section{Beasley $\mathrm{R}$ et al. Association between} paracetamol use in infancy and childhood, and risk of asthma, rhinoconjunctivitis, and eczema in children aged 6-7 years: analysis from Phase Three of the ISAAC programme. Lancet 2008; 372: 1039-48

\section{Asthmakontrolle in anderen Umständen}

\section{Beinträchtigt ein ungenügend kontrolliertes Asthma während der Schwangerschaft die Entwicklung des Fötus? Jetzt gibt es konkrete Belege speziell für die Frühschwangerschaft.}

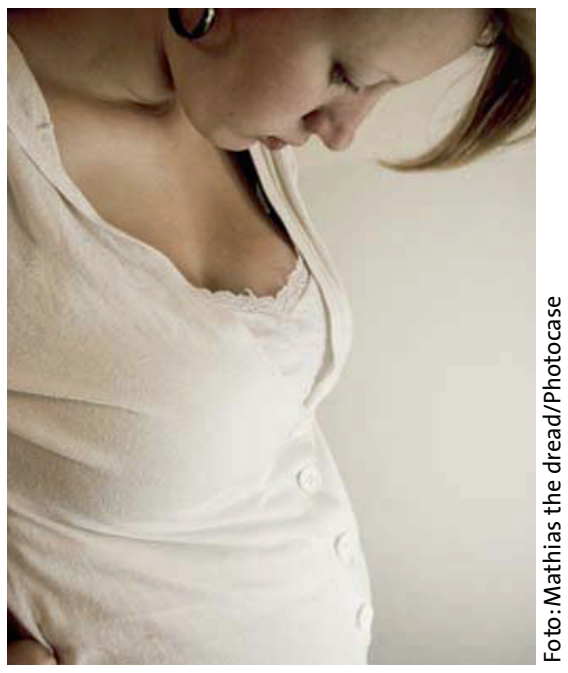

Asthma-Basismedikamente weiter anwenden: Sie schaden dem ungeborenen Kind weniger als eine Exazerbation! etrospektiv werteten Forscher aus Quebec, Kanada, die Daten von 4.344 Schwangerschaften bei Asthmapatientinnen im Hinblick auf die medizinische Betreuung im ersten Trimenon sowie auf den Gesundheitszustand der Neugeborenen aus.

In der Kohorte war fast jedes zehnte Kind (9,2\%) von mindestens einer Missbildung betroffen, etwa zwei Drittel der Fälle mussten als schwer eingestuft werden. Besonders häufig waren Bewegungsapparat und Herz betroffen. Babys von Frauen mit Asthmaexazerbationen im ersten Schwangerschaftstrimenon waren häufiger betroffen als Kinder von Frauen mit gut kontrolliertem Asthma (12,8 vs. $8,9 \%)$. Unter Berücksichtung anderer Einflussfaktoren wie Alter oder chronischer Erkrankungen ergab sich eine um 48\% erhöhte Wahrscheinlichkeit einer Fehlbildung bei schlecht kontrolliertem Asthma gegenüber einer Schwangerschaft ohne Exazerbation (Odds Ratio 1,48). Bezogen auf schwerwiegende Fehlbildungen war das Risiko durch Exazerbationen im ersten Trimester um $32 \%$ erhöht (Odds Ratio 1,32). Das erhöhte Risiko war nicht durch die Einnahme oraler Glukokortikoide zu erklären: Frauen mit Exazerbationen, die keine oralen Glukokortikoide erhalten hatten, brachten doppelt so häufig ein Kind mit Fehlbildungen auf die Welt wie Patientinnen ohne Exazerbationen in der Frühschwangerschaft.

Fazit: Diese retrospektive Kohortenstudie weist deutlich auf die Notwendigkeit einer guten Asthmakontrolle während der Frühschwangerschaft hin, um Fehlbildungen bei Kindern zu vermeiden. $f k$

Blais L et al. Asthma exacerbations during the first trimester of pregnancy and the risk of congenital malformations among asthmatic women. J Allergy Clin Immunol 2008; 121: 1379-84 